\title{
Positive hysteresis of Ce-doped GAGG scintillator
}

Takayuki Yanagida $^{1 *}$, Yutaka Fujimoto ${ }^{1}$, Masanori Koshimizu ${ }^{2}$, Kenichi Watanabe ${ }^{3}$, Hiroki Sato $^{4}$, Hideki Yagi ${ }^{5}$, Takagimi Yanagitani ${ }^{5}$

${ }^{1}$ Kyushu Institute of Technology, 2-4 Hibikino, Wakamatsu-ku, Kitakyushu 808-0196, Japan

${ }^{2}$ Tohoku University, 6-6-07 Aoba, Aramaki, Aoba-ku, Sendai 980-8579, Japan

${ }^{3}$ Nagoya University, Furocho, Chikusa, Nagoya, 464-8603 Japan

${ }^{4}$ Furukawa 1-25-13, Kannondai, Tsukuba, Ibaraki, 305-0856, Japan

${ }^{5}$ Konoshima Chemical Co., Ltd., 80 Kouda, Takuma, Mitoyo, Kagawa 769-1103, Japan

\begin{abstract}
Positive hysteresis and radiation tolerance to high-dose radiation exposure were investigated for $\mathrm{Ce} 1 \%$ and $3 \%$ doped $\mathrm{Gd}_{3}(\mathrm{Al}, \mathrm{Ga})_{5} \mathrm{O}_{12}(\mathrm{Ce}: \mathrm{GAGG})$ crystal scintillator on comparison with other garnet scintillators such Ce:YAG, Ce:LuAG, Pr:LuAG, and ceramic Ce:GAGG. When they were irradiated by several Gy ${ }^{60} \mathrm{Co} \gamma$-rays, Ce $1 \%$ doped GAGG crystal exhibited $~ 20 \%$ light yield enhancement (positive hysteresis). This is the first time to observe positive hysteresis in Ce doped GAGG. On the other hand, other garnet materials did not show the positive hysteresis and their light yields were stable after 800 Gy irradiation except Pr:LuAG. The light yield of Pr:LuAG decreased largely. When irradiated Ce:GAGG which showed positive hysteresis was evaluated in Synchrotron facility (UVSOR), new excitation band was created around $60 \mathrm{~nm}$.
\end{abstract}

Keywords: $\quad$ single crystal;ceramic; Scintillation detector; $\mathrm{Ce}^{3+} ; \mathrm{Pr}^{3+} ;$ Scintillator; positive hysteresis

PACS: $07.57 . \mathrm{K}, 78.60 . \mathrm{Y}, 78.20$

* Corresponding author. Present address: Kyushu Institute of Technology, 2-4 Hibikino, Wakamatsu-ku, Kitakyushu 808-0196, Japan Tel.: +8193695 6049; fax: +81936956049. E-mail address: yanagida@1sse.kyutech.ac.jp (Takayuki Yanagida) 


\section{Introduction}

Inorganic scintillators which convert high energy ionizing radiation to thousands of UVvisible photons [1], have been playing a major role in many fields of radiation detection, including medical imaging [2], security [3], astrophysics [4], particle physics [5], and welllogging [6]. In these applications, scintillators for $\gamma$-ray detectors have attracted much attention because of the large volume of market especially in medical and security applications. Radiation tolerance is one of the important properties for scintillators and many materials were examined in this property. In radiation tolerance experiments, one interesting phenomenon, positive hysteresis was discovered. Positive hysteresis is a phenomenon in which the light yield of the scintillator is increased after exposure to ionizing radiation [7]. At present, Ce-doped $\mathrm{Gd}_{2} \mathrm{SiO}_{5}$ (Ce:GSO) [8] and Tl-doped CsI (Tl:CsI) [9] showed positive hysteresis. The origin of positive hysteresis is still under discussion, but there are some known experimental results that positive hysteresis is a temporary phenomenon and continues few days depending on the irradiated dose.

The aim of this work is to examine radiation tolerance and positive hysteresis of famous garnet scintillators, including crystal $\mathrm{Ce} 1 \%$ and $3 \%$ doped $\mathrm{Gd}_{3}(\mathrm{Al}, \mathrm{Ga})_{5} \mathrm{O}_{12}(\mathrm{Ce}: \mathrm{GAGG})$ [10], Ce $0.5 \%$ doped $\mathrm{Y}_{3} \mathrm{Al}_{5} \mathrm{O}_{12}$ ceramic (Ce:YAG) [11], Ce $0.5 \%$ doped $\mathrm{Lu}_{3} \mathrm{Al}_{5} \mathrm{O}_{12}$ (Ce:LuAG) ceramic [12], Pr 0.25\% doped LuAG (Pr:LuAG) [13], and ceramic Ce 1\% doped GAGG [14]. Crystalline scintillators were fabricated by the conventional Czochralski method in Furukawa and ceramic ones were prepared by the vacuum sintering in Konoshima Chemical, respectively. Among above luminescent materials, crystal Ce:GAGG and Pr:LuAG by Furukawa are commercially available for scintillator uses, and ceramic Ce:YAG by Konoshima is a product for general phosphor applications. Figure 1 shows appearances of samples for present study. Most samples had thin plate shape while Pr:LuAG had rectangular shape because it was fabricated for Positron Emission Mammography [2]. 


\section{Experimental procedures}

First, optical in-line transmittance was evaluated by using V670 spectrometer (JASCO) before ${ }^{60} \mathrm{Co} \gamma$-ray evaluation since it was a general way to evaluate radiation tolerance on a degradation of transmittance. Photoluminescence (PL) quantum yield $(Q Y)$ was also evaluated using a Hamamatsu Quantaurus-QY. The absolute PL $Q Y$ was calculated by the following equation, $Q Y=N_{\text {emit }} / N_{\text {absorb }}$, where $N_{\text {emit }}$ and $N_{\text {absorb }}$ are the numbers of emission and absorption photons, respectively [15]. These properties were also evaluated after $800 \mathrm{~Gy}{ }^{60} \mathrm{Co}$ $\gamma$-ray exposure. Because our laboratory and the irradiation facility were not so close, these basic optical properties were evaluated $6,24,48$, and 72 hours after the irradiation.

Then, basic scintillation properties such as X-ray-induced radioluminescence spectra and scintillation decay time profiles were evaluated at the Kyushu Institute of Technology to confirm that our materials were not specific ones. X-ray-induced emission spectra were measured using a DU420A-BU2 Charge Coupled Device (CCD) camera equipped with an SR163 monochromator, made by Andor Technology. The CCD was cooled to $188 \mathrm{~K}$ using a Peltier module. The excitation source was an X-ray generator supplied with $80 \mathrm{kV}$ and $1 \mathrm{~mA}$. The scintillation emission was fed to the monochromator through a $2 \mathrm{~m}$ optical fiber to avoid X-rays hitting the CCD directly. A detailed explanation of the radioluminescence setup was previously published [16]. Scintillation decay time profiles were evaluated using our original setup, a pulse X-ray streak camera system also described previously [17-18].

In the irradiation facility of Nagoya Univ., $800 \mathrm{~km}$ distant from the Kyushu Institute of Technology, ${ }^{60} \mathrm{Co}$ was used to irradiate samples in steps of $200 \mathrm{~Gy}$ from 0 to $800 \mathrm{~Gy}$. At each step, we evaluated the pulse height spectrum using a ${ }^{137} \mathrm{Cs}$ source. As described later, Ce $1 \%$ doped GAGG crystal exhibited the positive hysteresis, and we irradiated smaller dose (1-20 Gy) to this sample to determine the threshold of the phenomenon. In pulse height 
measurements, we mounted each crystal on the PMT (R7600-200, Hamamatsu) with silicon grease (OKEN 6262A). When $\gamma$-rays from ${ }^{137} \mathrm{Cs}$ were detected, the signals were fed into a preamplifier (ORTEC 113), a shaping-amplifier (ORTEC 572) with $2 \mu$ s shaping time, a multichannel analyzer (hereafter MCA, Kromek K102), and finally to a personal computer. The time interval between the ${ }^{60} \mathrm{Co}$ exposure and pulse height spectrum measurement was typically a few minutes, the time required to walk from the irradiation room to the measurement room in the same building. All experiments were carried out at room temperature.

Finally, excitation bands in higher energy range $(50-200 \mathrm{~nm})$ of Ce $1 \%$ doped GAGG was evaluated in Synchrotron facility (UVSOR). In this experiment, we prepared two samples and one of them was irradiated with 2000 Gy at Nagoya Univ. After one of our colleagues took the sample from Nagoya Univ. to UVSOR, we compared excitation bands of irradiated and non-irradiated Ce $1 \%$ doped GAGG samples. The time interval of transportation from Nagoya Univ. to UVSOR was around 1.5 hours. In UVSOR, we used BL-7B beam line and the measurements were carried out at room temperature.

\section{Results and Discussion}

\subsection{Basic scintillation characteristics before the irradiation}

Figure 2 represents normalized X-ray excited radioluminescence (scintillation) spectra of present samples. Around $520 \mathrm{~nm}$ emission in Ce-doped materials was ascribed to $\mathrm{Ce}^{3+} 5 \mathrm{~d}-4 \mathrm{f}$ transition and $300 \mathrm{~nm}$ emission in Pr:LuAG was due to $\operatorname{Pr}^{3+} 5 \mathrm{~d}-4 \mathrm{f}$ transition, respectively. In ceramic Ce:GAGG emission around $380 \mathrm{~nm}$ was arized from impurity (perovskite) phase as discussed previously [14]. Since crystal field affects $5 \mathrm{~d}$ levels of materials, emission peaks in Ce-doped materials differed. Then, scintillation decay time profiles of garnet scintilaltors are demonstrated in figure 3. All Ce-doped scintillators exhibited $\sim 100$ ns primary decay time 
while that of Pr:LuAG was $~ 20$ ns. These values were typical for $\mathrm{Ce}^{3+}$ and $\operatorname{Pr}^{3+} 5 \mathrm{~d}-4 \mathrm{f}$ transition. Through out these evaluations, our samples were not specific materials in scintillation characteristics.

\subsection{Radiation tolerance}

First, transmittance spectra of samples in this work were investigated. Consequently, no significant differences were observed after the ${ }^{60} \mathrm{Co} \gamma$-ray irradiation. Recently, radiation damage of Pr:LuAG was studied and transmittance of Pr:LuAG degraded largely in high dose irradiation [19]. In several hundreds Gy exposure by the present work, no transmittance degradation occured. Compared with Ce:GSO studied recently which showed transmittance degradation [8], garnet scintillators had high resistivity to radiation exposure in optical transmittance.

Figure 4 demonstrates pulse height spectra of Ce 1\% doped GAGG from 0 to $20 \mathrm{~Gy}$ exposure and Pr:LuAG from 0 to $600 \mathrm{~Gy}$. After 800 Gy irradiation, we could not detect signal from Pr:LuAG due to a degradation of light yield and a huge phosphorescence in lower MCA channels. As described later, other samples were stable in pulse height spectra. Ce $1 \%$ doped GAGG showed positive hysteresis and the light yield was enhanced $\sim 20 \%$. This tendency did not change to $800 \mathrm{~Gy}$ irradiation in this sample. We investigated 1-3 and 20 Gy exposure so that the threthold of positive hysteresis in Ce:GAGG was around $10 \mathrm{~Gy}$. It was the first time to observe positive hysteresis in Ce:GAGG. On the other hand, Pr:LuAG exhibited a huge degradation of light yield and it was consistent with recent result that Pr:LuAG was not so resistive to high dose exposure [19]. Though it was not a main object of this project, the reason of worse energy resolution of Pr:LuAG before the irradiation was blamed for the optical attachement. To measure all samples quickly, we attached the wide surface of the sample to PMT and light collection efficiency was not so good in this experiment. Figure 5 
summarizes normalized scintillation light yield in pulse height after irradiation. In this figure, light yield (662 keV photoabsorption peak channel) before the irradiation was defined to 1 . Except Ce 1\% doped GAGG and Pr:LuAG, scintillation light yield was stable. In Ce-doped GAGG samples, low Ce concentration sample exhibited positive hysteresis and the tendency was same with the case of Ce:GSO [8]. Taking into account the segregation coefficient of crystal Ce:GAGG [10], actual doping concentrations of Ce $1 \%$ and $3 \%$ doped crystals were 0.45 and $1.2 \%$, respectively. As the ceramic is free from the segregation in macroscopic scale, actual Ce concentrations were Ce $1 \%$ crystal < Ce $1 \%$ ceramic < Ce 3\% crystal. It must be noted that ceramic Ce:GAGG had the highest Ce concentration among GAGG samples because it was fabricated by the solid state reaction and free from the segeregation which was not avoidable in conventional melt growth.

In scintillation, luminescence efficiency were simply devided to two kinds; one is a transfer from host to emission centers (mainly outside of the band gap) and efficiency at luminescence center (inside the band gap). Simple formula, $n_{\mathrm{e}-\mathrm{h}}=B E_{\gamma} / \beta E_{\mathrm{g}}$, was raised [20-23]. In this formula, $E_{\gamma}$ is the energy deposited by $\gamma$-quanta, The factor $B$ relates the phonon energy loss during the ionization and is expressed as $B=1 /(1+0.65 K)$, where $K$ is defined as the ratio of energy lost by optical phonons to the energy lost for an ionization event [24]. Thus, the light yield $(L Y)$ is simply expressed as $L Y=10^{6} \mathrm{~S} Q B / \beta E_{\mathrm{g}}[\mathrm{ph} / \mathrm{MeV}]$, where $S$ is the energy transport efficiency from the host to the emission center, $Q$ is the luminescence quantum efficiency at the emission center which equals to $Q Y$ in this work, and $\beta$ is an empirical constant close to 2.5 in famous scintillators and is theoretically 2.3. Thus to understand the phenomenon, separation of $B, S$, and $Q$ is important. Figure 6 shows $Q Y$ of present samples before ( 0 hour) and after $(6,24,48,72$ hours) irradiation. Ce:LuAG exhibited an interesting change that the degradation and recovery. In Pr:LuAG, $Q Y$ decreased from $92 \%$ to $40 \%$ and it did not recover at least within 72 hours. Therefore, 90\% light yield degradation of Pr:LuAG was ascribed to 
the degradation of both $Q Y$ and $B S$. On the other hand, other Ce-doped garnet scintillators did not show significant changes and it meant that the positive hysteresis of Ce $1 \%$ doped GAGG was mainly caused by the change of $B S$.

Figure 7 represents excitation spectra monitoring at 500-650 $\mathrm{nm}$ integrated counts of irradiated and non-irradiated Ce 1\% doped GAGG. Around $200 \mathrm{~nm}, 160$, and $80 \mathrm{~nm}$ peaks were detected in both samples. The $200 \mathrm{~nm}$ band corresponded to band gap of GAGG host and $160 \mathrm{~nm} 80 \mathrm{~nm}$ bands would be due to $5 \mathrm{~d}_{4}$ and $5 \mathrm{~d}_{5}$ levels of $\mathrm{Ce}^{3+}$ because $5 \mathrm{~d}_{1-3}$ bands appeared around 460, 340, and $230 \mathrm{~nm}$ in Ce-doped garnet materials. In the irradiated sample, new excitation band around $60 \mathrm{~nm}$ was created. $60 \mathrm{~nm}$ is close to $2.5 \times E_{\mathrm{g}}$ (see above formula) which corresponded to typical average energy to create one electron-hole pair in materials under high energy ionizing radiation exposure and such energy would have some physical meanings.

Though we did not conclude this newly created band fully or partially affected positive hysteresis by presented data sets, it would relate the phenomenon. Based on the meta-stable characteristics of the positive hysteresis, it is reasonable to consider that irradiation-induced meta-stable centers are involved in the scintillation process after irradiation. In the previous paragraph, we mentioned that the positive hysteresis is due to the change in $B S$. In the following, we propose two possible mechanisms for the change in $B$ or $S$ and the appearance of the excitation band at $60 \mathrm{~nm}$.

The change in $B$ would be caused by the change in the electronic band structure due to the creation of the metastable centers. The change in the band structure may lead to the change in the fraction of the energy loss via phonon emission, because this fraction is related to the electronic energy structure [25]. In this case, the positive hysteresis is explained in terms of the decrease in the energy loss fraction. In this scenario, the appearance of the $60 \mathrm{~nm}$ excitation band is explained as follows: Because the photon energy at $60 \mathrm{~nm}$ is close to the 
average energy for electron-hole pair creation, the number of electron-hole pairs created by a single VUV photon is close to unity down to $60 \mathrm{~nm}$, and electron-hole pair multiplication sets in for shorter wavelength. If we assume the decrease in the energy loss fraction, it is reasonable to consider that the probability of the multiple electron-hole pairs would be enhanced at $60 \mathrm{~nm}$. Thus, the positive hysteresis and the appearance of the excitation band at $60 \mathrm{~nm}$ can be explained in a consistent manner.

On the other hand, a scenario for the change in $S$ is the change in the energy transfer process. In this scenario, the meta-stable centers are assumed to promote the energy transfer to $\mathrm{Ce}^{3+}$. Considering the appearance of the excitation band at $60 \mathrm{~nm}$, this promotion is effective particularly for the electron-hole pairs having a relatively higher energy, because the photon energy at $60 \mathrm{~nm}$ is significantly higher than the bandgap energy of GAGG. One possible explanation in this line is that the meta-stable centers change the relaxation path of such high-energy electron-hole pairs, which leads to some relaxed states preferable to the energy transfer to $\mathrm{Ce}^{3+}$.

Most probably oxygen vacancy was created by $\gamma$-ray irradiation and such vacancies temporary created meta-stable level beyond the band gap of the material. If this high energy excitation band is profoundly related to the origin of the positive hysteresis, it has a big merit for understanding and engineering scintillation light yield of materials through elaboration of the high-energy electronic band structure.

\section{Conclusion}

Crystal Ce $1 \%$ and 3\% doped GAGG, Pr:LuAG, ceramic Ce:YAG, Ce:LuAG, and Ce:GAGG were investigated on their basic scintillation characteristics and radiation tolerance. Among them, Ce 1\% doped GAGG crystal exhibited positive hysteresis and the light yield was enhanced $20 \%$. This is the first time to observe positive hysteresis in Ce doped GAGG. 
The radiation resistivity of Pr:LuAG was not so high compared with other oxide scintillators. Other materials, Ce 3\% doped GAGG, Ce:YAG, Ce:LuAG, and ceramic Ce:GAGG was highly resistive to ionizing radiation bombardment.

\section{Acknowledgments}

This work was mainly supported by JST Sentan, A-step and partially by a Grant in Aid for Young Scientists (A)-23686135, and Challenging Exploratory Research-23656584 from the Ministry of Education, Culture, Sports, Science and Technology of the Japanese government (MEXT). Partial assistance from the Yazaki Memorial Foundation for Science and Technology, Shimazu Sci. Foundation, Kato Foundation for Promotion of Science, and Nippon Sheet Glass Foundation for Materials Science and Engineering, Tokuyama Science foundation, Iketani Science and Technology Foundation, Hitachi Metals Materials Science Foundation, Mazda Foundation, JFE $21^{\text {st }}$ century Foundation, and The Asahi Glass Foundation, are also gratefully acknowledged. 


\section{References}

[1] T. Yanagida, Opt. Mater., 351987 (2013).

[2] T. Yanagida, A. Yoshikawa, Y. Yokota, K. Kamada, Y. Usuki, S. Yamamoto, M. Miyake, M. Baba, K. Sasaki, M. Ito, IEEE. Nucl. Trans. Sci. 571492 (2010).

[3] D. Totsuka, T. Yanagida, K. Fukuda, N. Kawaguchi, Y. Fujimoto, Y. Yokota, A. Yoshikawa, Nucl. Instrum. Meth A 659399 (2011).

[4] K. Yamaoka, M. Ohno, Y. Terada, S. Hong, J .Kotoku, Y. Okada, A. Tsutsui, Y. Endo, K. Abe, Y. Fukazawa, S. Hirakuri, T. Hiruta, K. Itoh, T. Itoh, T. Kamae, M. Kawaharada, N. Kawano, K. Kawashima, T. Kishishita, T. Kitaguchi, M. Kokubun, G.M. Madejski, K. Makishima, T. Mitani, R. Miyawaki, T. Murakami, M.M. Murashima, K. Nakazawa, H. Niko, M. Nomachi, K. Oonuki, G. Sato, M. Suzuki, H. Takahashi, I. Takahashi, T. Takahashi, S. Takeda, K. Tamura, T. Tanaka, M. Tashiro, S. Watanabe, T. Yanagida, D. Yonetoku, IEEE. Trans. Nucl. Sci, 52, 2765 (2005).

[5] T. Ito, T.Yanagida, M. Sato, M. Kokubun, T. Takashima, S. Hirakuri, R. Miyawaki, H. Takahashi, K. Makishima, T. Tanaka, K. Nakazawa, T. Takahashi, T. Honda, Nucl. Instrum. Meth. A 579239 (2007).

[6] T. Yanagida, Y. Fujimoto, S. Kurosawa, K. Kamada, H. Takahashi, Y. Fukazawa, M. Nikl, V. Chani, Jpn. J. Appl. Phys. 52 (2013) 076401.

[7] M. Tanaka, K. Hara, S. Kim, K. Kondo, H. Takano, H. Kobayashi, H. Ishibashi, K. Kurashige, K. Susa, M. Ishii, Nucl. Instr. Meth-A 404283 (2002).

[8] T. Yanagida, Y. Fujimoto, K. Watanabe, Radiat. Meas., accepted (2013).

[9] C. Grescovich, D. Cusano, D. Hoffman, R. J. Riedner, Am. Ceram. Soc. Bull. 71, 1120 (1992).

[10] K. Kamada, T. Yanagida, J. Pejchal, M. Nikl, T. Endo, K. Tsutsumi, Y. Fujimoto, A. Fukabori, A. Yoshikawa, IEEE Trans. Nucl. Sci., 59 2112-2115 (2012). 
[11]H. Takahashi, T. Yanagida, D. Kasama, T. Ito, M. Kokubun, K. Makishima, T. Yanagitani, H. Yagi, T. Shigeta, T. Ito, IEEE Trans. Nucl. Sci., 53, 2404-2408 (2006).

[12]T. Yanagida, Y. Fujimoto, Y. Yokota, K. Kamada, S. Yanagida, A. Yoshikawa, H. Yagi, T. Yanagitani, Radiat. Meas., 46 1503-1505 (2011).

[13] T. Yanagida, M. Sato, K. Kamadaa, Y. Fujimoto, Y. Yokota, A. Yoshikawa, V. Chani, Opt. Mater., 33413 (2011).

[14] T. Yanagida, K. Kamada, Y. Fujimoto, H. Yagi, T. Yanagitani, Opt. Mater., 352480 (2013).

[15] T. Yanagida, Y. Fujimoto, K. Fukuda, V. Chani, Nucl. Instrum. Methods A, 72958 (2013).

[16] T. Yanagida, N. Kawaguchi, Y. Fujimoto, K. Fukuda, K. Watanabe, A. Yamazaki, A. Uritani, J. Lumin, 144 212-216 (2013).

[17] T. Yanagida, Y. Fujimoto, A. Yoshikawa, Y. Yokota, K. Kamada, Jan Pejchal, N. Kawaguchi, K. Fukuda, K. Uchiyama, K. Mori, K. Kitano, M. Nikl, Appl. Phys. Express, 3056202 (2010).

[18]T. Yanagida, Y. Fujimoto, A. Yamaji, N. Kawaguchi, K. Kamada, D. Totsuka, K. Fukuda, K. Yamanoi, R. Nishi, S. Kurosawa, T. Shimizu, N. Sarukura, Radiat. Meas., 5599 (2013).

[19]M.V. Derdzyan, K.L.Ovanesyan, A.G.Petrosyan, A.Belsky, C.Dujardin, C.Pedrini, E. Auffray, P.Lecoq, M.Lucchini, K.Pauwels, J. Cryst. Growth, 361212 (2012).

[20]D. J. Robbins, J. Electrochem. Soc. 127, 2694 (1980).

[21]P. Dorenbos, J. Lumin, 91, 91 (2000).

[22] A. Lempicki, A.J. Wojtowicz, E. Berman, Nucl. Instrum. Meth. A 333304 (1993).

[23] G. Blasse, B. C. Grabmaier, Luminescent Materials (Berlin: Springer) (1994).

[24] V. B. Mikhailik, H. Kraus, Phys. Status Solidi B 247, 1583-1599 (2010). 
[25] W. Shockley, Kittel, Phys. Rev. 80, 6 (1950). 
Figure Captions

Figure 1 Appearance of sample scintillators.

Figure $2 \mathrm{X}$-ray induced radioluminescence spectra of sample scintillaors.

Figure 3 Scintillation time profiles of garnet scintillators under pulse X-ray excitation.

Figure $4{ }^{137}$ Cs pulse height spectra of Ce 1\% doped GAGG crystal (left) and Pr:LuAG (right) after several dose irradiation.

Figure 5 Normalized scintillation light yield after 0-800 Gy X-ray exposure.

Figure 6 PL $Q Y$ after 800 Gy X-ray exposure. Measurements were done 6, 24, 48, 72 hours later finishing the irradiation.

Figure 7 Excitation spectra of irradiated (dotted line) and non-irradiated (solid line) Ce 1\% doped GAGG crystal. The monitoring wavelength was integrated from 500 to $650 \mathrm{~nm}$. 
Ce $1 \%$ GAGG (Furukawa)

Ce 3\% GAGG (Furukawa)

Ce 1\% GAGG (Konoshima)

Ce $0.5 \%$ YAG (Konoshima)

Ce $0.5 \%$ LuAG (Konoshima)

Pr 0.25\% LuAG (Furukawa)

0

10

20

30

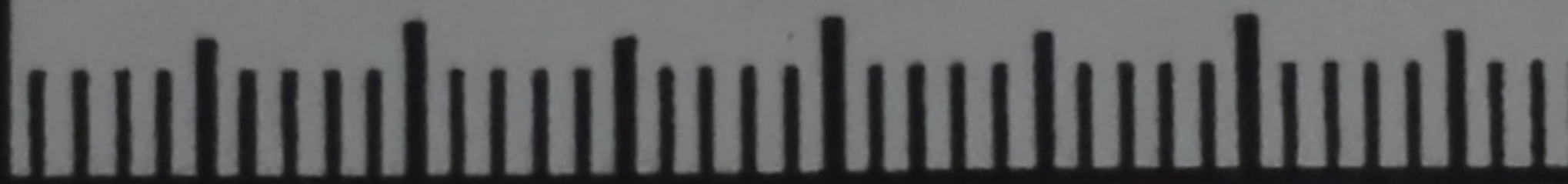


Figure2

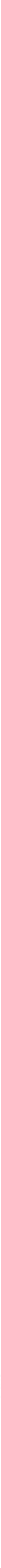




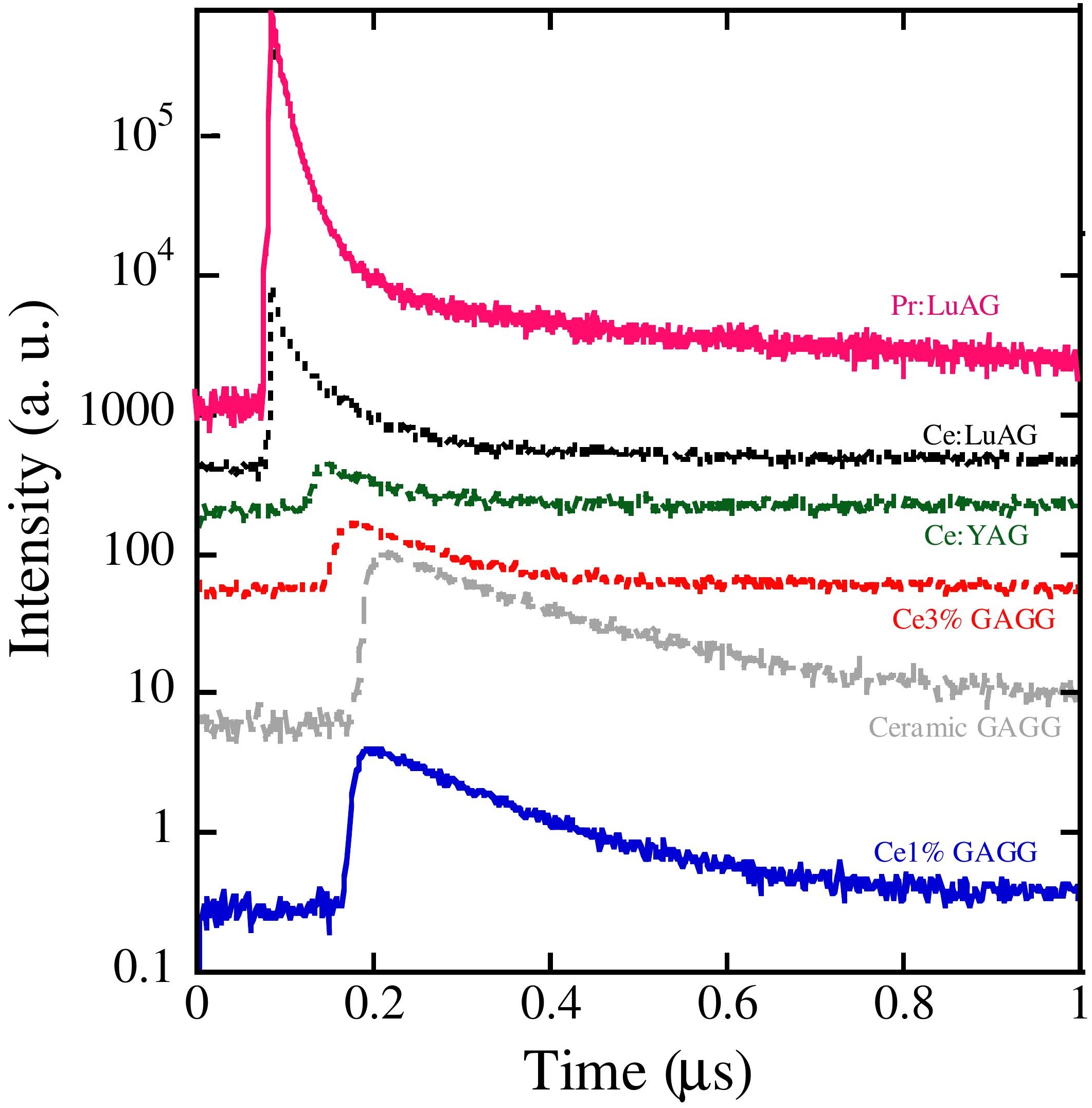




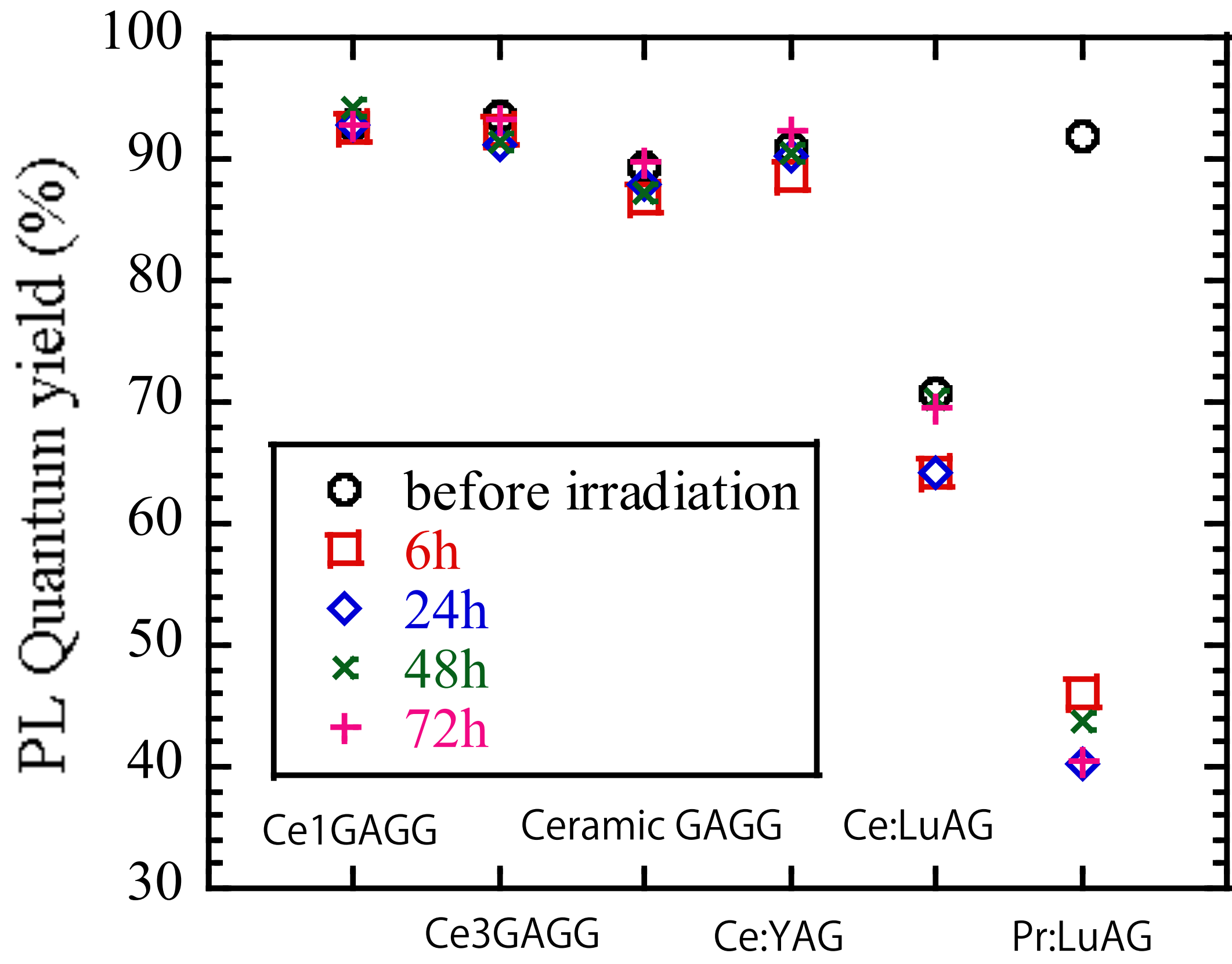

\section{Ce3GAGG Ce:YAG Pr:LuAG}




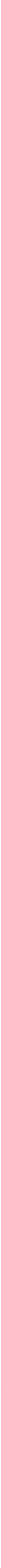

Figure7
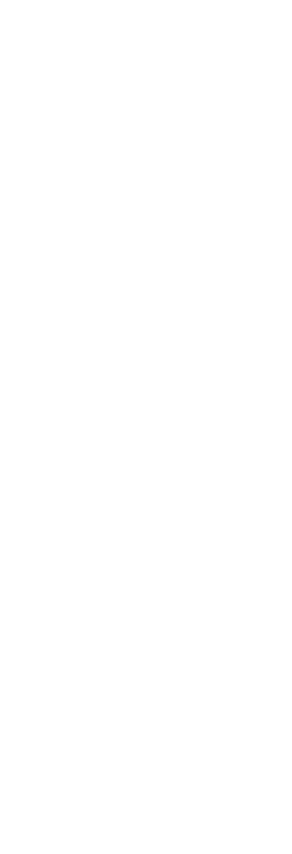

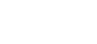

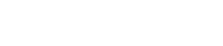

.

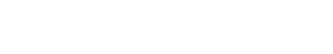

.

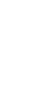

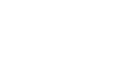$$
\text { . }
$$ 